\title{
Presentación
}

\section{PENSAR LAS TRANSFORMACIONES DEL COMPROMISO Y DE LA PARTICIPACIÓN POLÍTICA}

Stéphanie Alenda*

Ante las evoluciones de las democracias representativas, uno de los primeros reflejos consiste en preocuparse por la desafección ciudadana hacia la cosa pública, el debilitamiento de los partidos políticos, la reducción de los efectivos militantes y el aumento de la abstención, a lo cual invitan los debates teóricos internacionales y nacionales sobre la cuestión (Dalton y Wattenberg, 2000; Ion, 1997; Torcal, 2003). Con el ocaso de las ideologías como telón de fondo, resulta también tentador pronosticar si no "el fin de lo político", al menos la "despolitización" o la "desmovilización social" de la que dieron cuenta varios estudios sobre la postdictadura chilena (Oxhorn, 1994; Hipsher, 1996; Schneider, 2000). Otros trabajos mostraron en cambio el carácter cíclico y variable de estas tendencias según los contextos ${ }^{1}$, lo que pareciera corroborar la actual ola de movilizaciones sociales en Chile, que contrasta con la atonía militante de los últimos años.

Estas expresiones de protesta levantan preguntas sobre los cambios democráticos e incitan a enfocar la participación política como un conjunto de comportamientos políticos tanto convencionales (instituciones políticas) como no convencionales (movimientos sociales). El planteamiento no es nuevo. Tras el cambio mayor de enfoque que conoció la sociología de la acción colectiva a partir de los años sesenta, en particular a raíz del movimiento por los derechos civiles en Estados Unidos y de las movilizaciones estudiantiles en varias partes del mundo, el estudio de los movimientos sociales empezó a ser relacionado con el poder político, con una revaluación del impacto de éstos sobre las instituciones políticas². Gracias a los notables aportes de la sociología anglosajona sobre los movimientos sociales, éstos dejaron de ser percibidos como formas apolíticas de comportamiento colectivo y fue cuestionada la demarcación entre el poder institucionalizado y el poder en movimiento. Perdió también sentido la separación entre los objetos circunscritos a la ciencia política y los que serían del exclusivo dominio de la sociología. Este cambio de perspectiva constituyó sin lugar a dudas un punto de inflexión para las investigaciones posteriores que buscaron

* Directora de la Escuela de Sociología de la Universidad Andrés Bello. Profesora asistente del Instituto de Asuntos Públicos de la Universidad de Chile y miembro del claustro del Doctorado en Ciencias Sociales de esta misma Universidad. Miembro del grupo internacional de trabajo sobre los Miembros y Militantes de los Partidos-Members and Activists of Political Parties (MAPP), patrocinado por la International Sociological Association (ISA) y la International Political Science Association (IPSA).

$1 \quad$ Respecto a las evoluciones de los efectivos de los partidos, se puede consultar a Rocha (2009) sobre el caso del PT o a Astrid Barrio et al. (2011) sobre los partidos españoles, entre varias otras referencias.

2 Véase, entre otras referencias, Doug McAdam (1982). 
pensar dentro de un continuo los comportamientos de participación, desde la contestación hasta las instituciones participativas, pasando por los sindicatos y los partidos.

Al abrir pistas para la comprensión de los cambios democráticos y de la participación, los artículos reunidos en el presente número sin duda contribuyen a enriquecer los debates contemporáneos sobre las renovaciones de la democracia, de máxima trascendencia en el Chile de hoy. Aunque tomen como objeto los partidos o los movimientos sociales, todos comparten una mirada sociológica sobre los fenómenos políticos, por definición comprensiva y sometida a las reglas de un método específico. Estos artículos tienen también en común el adscribirse a un postulado constructivista inicialmente inspirado en la sociología de Max Weber y de Pierre Bourdieu. Cabe enfatizar que esta mirada se forjó a contracorriente de las tendencias paradigmáticas de la ciencia política actual, más macroscópicas, adictas a las modelizaciones, al tratamiento a distancia del objeto, o a la defensa irrestricta de una pureza disciplinaria que no resiste la observación empírica ni la necesidad de dar cuenta de fenómenos complejos que precisan entradas múltiples.

Más específicamente, los siete artículos que componen este número constituyen aportes para la reflexión sobre las nuevas formas de militancia y de participación en el espacio público. Dos de ellos, el de Boris Gobille/Eric Agrikoliansky y de Ivan Bruneau, analizan el movimiento altermundialista, presentado por los dos primeros autores como la "Internacional de los excluidos y de las minorías para defenderse contra la Internacional de las élites que no dice su nombre". Mientras su trabajo ofrece un análisis del activismo altermundialista en Europa, el texto de Bruneau restituye el proceso a raíz del cual un sindicato agrícola francés, la Confederación Campesina, convergió hacia el movimiento antiglobalización en 1999.

Ambos artículos permiten recordar que, pese a tener una coordinación transnacional, no debe ser sobrestimada la capacidad de autonomización del movimiento respecto a las influencias estatales nacionales y locales directas; tampoco debe serlo su "novedad" compartida por formas de acción colectiva anteriores tales como las Internacionales comunistas o Greenpeace. La "transnacionalización" de la protesta (Tarrow, 2000; Della Porta y Tarrow, 2005) aparece así más reivindicada que real. Es al menos lo que pone en evidencia la reinscripción de estos movimientos en las configuraciones nacionales, regionales e incluso locales en las que emergen así como la consideración del tipo de evento que congrega a los activistas. Gobille y Agrikoliansky analizan el altermundialismo "como el resultado de la dinámica misma de los eventos de protesta", recurriendo a los resultados de dos encuestas inspiradas en el "Individual Survey in Rallies" y dirigidas a activistas europeos en 2003, en la contracumbre del G8 en la frontera franco-suiza y durante el Foro Social Europeo (región parisina). Si bien el perfil de los participantes corresponde al de jóvenes pertenecientes a una élite social, dotada por lo general de un fuerte capital cultural y de estabilidad laboral, por lo demás atraída por estructuras de participación flexibles, sus propiedades sociales varían levemente de un evento a otro. Por su parte, Bruneau recurre al análisis localizado para demostrar las condiciones del éxito de la acción de "desmontaje" de un McDonald's, suceso a raíz del cual la lucha sindical de la Confederación Campesina desemboca en la causa altermundialista. 
Desde el punto de vista organizacional, la "flexibilización" del movimiento altermundialista parece deber mucho a su dimensión internacional. Éste adopta en efecto una estructura en redes que toma la forma de una nebulosa de pequeñas unidades (ONGs, sindicatos, partidos políticos, órganos de difusión como Le Monde Diplomatique, a su vez impulsor de ATTAC en 1997) relacionadas entre sí de forma dúctil y conectadas esporádicamente para llevar a cabo acciones puntuales. Como muestra Bruneau, estas redes "de relaciones militantes, formales e informales" operan también a nivel local e invitan a estudiar dicho movimiento como "el producto de filiaciones activistas y organizacionales anteriores" (Gobille, Agrikoliansky), principalmente sindicales y asociativas pero también partidistas, lo que pone de nuevo en entredicho su "novedad".

Fruto de tradiciones militantes marginalizadas (Agrikoliansky et al., 2005) y de organizaciones sindicales minoritarias tales como la Confederación Campesina de José Bové analizada por Bruneau, el altermundialismo encarna la posibilidad de salir del letargo militante, reciclando viejas ideologías y pertenencias previas. Su organización descansa en gran medida en un "activismo experto" (Collovald et al., 2003), reflejado en el alto nivel de formación universitaria que caracteriza a sus protagonistas (Gobille y Uysal, 2005). La perspectiva genealógica que adoptan ambos autores no sólo permite aprehender las filiaciones del movimiento (tercermundismo, ecologismo...) y las reconversiones de sus miembros, sino también las recomposiciones del espacio de los movimientos sociales desde los años ochenta (Gobille, Agrikoliansky) o las del registro protestatario construido durante los años setenta-ochenta (Bruneau) en Francia. Una de las interrogantes que levantan estos estudios para los países del sur es por qué este modelo de acción colectiva no ha prendido mayormente, pese a su vocación de "pensar global", y de ser la voz de las naciones más postergadas. En el caso de Chile, ciertas temáticas y formas de acción altermundialistas han permeado tanto las movilizaciones en contra de Hidroaysen ${ }^{3}$ en mayo de 2011, como las demandas estudiantiles, con la aparición de eslóganes como "Otro Chile es posible". Sería sin embargo apresurado deducir de aquello un giro en la historia del conflicto social nacional.

Cinco de los siete artículos reunidos en este número tratan también de los partidos políticos y la militancia, objeto sobre el cual este enfoque sociológico ha sido particularmente prolífico e innovador al realizar una síntesis entre diferentes teorías y herramientas de análisis, desde la sociología clásica (i.e. el partido como "relación social" y "empresa política" de Weber) hasta la sociología de los campos (Bourdieu). Las investigaciones más recientes sobre el tema buscaron también asociar y no oponer la sociología de las carreras (Becker, Hugues) y de las trayectorias biográficas (Bourdieu) para dar cuenta de lógicas sociales y personales de compromiso. Se alimentaron además de los marcos teóricos de la sociología de la acción colectiva para pensar las formas de movilización partidista y la participación política en general, contribuyendo asimismo a la superación del hiato entre el estudio de los comportamientos políticos convencionales y no convencionales.

3 Polémico proyecto que contempla la construcción y operación de varias centrales hidroeléctricas, ubicadas en la región de Aysén, en el sur de Chile. 
Dos rasgos centrales se desprenden de estos cinco trabajos: primero, una preocupación por reencastrar los partidos en su contexto sociohistórico y espacial; segundo, el planteamiento de no desvincular el análisis de las formas organizacionales, prácticas militantes o estrategias políticas de las propiedades sociales del personal partidista, de sus trayectorias y "multiposicionalidad" (Boltanski, 1973). En el artículo "Para una sociología de los entornos y de las redes partidistas", si bien Frédéric Sawicki reconoce que la autonomización parcial del campo político a raíz de la profesionalización política "justifica plenamente el estudio, de manera autónoma, de la organización de los partidos y de las estrategias que nacen de su participación en el campo político (...)", parte cuestionando la tendencia reificadora y homogeneizadora de las tipologías que aparecen como la finalidad principal de numerosos estudios. Éstas suelen en efecto ignorar que los partidos políticos emergen y se desarrollan en determinadas sociedades nacionales, y que provienen además de grupos específicos. En la línea de Michel Offerlé (1987), el autor aboga más bien por un enfoque en términos de "construcción social de las organizaciones partidarias" recurriendo al análisis de redes para aprehender "el entramado de las relaciones cotidianas" observables en los diferentes espacios de sociabilidad (asociaciones, sindicatos, empresas, cafés, etc.) que conforman el "entorno partidista". Una de las virtudes del artículo radica en ofrecer un método para comprobar que la unicidad de las etiquetas partidistas suele esconder formas variadas de arraigo local, lo que el autor muestra en el caso del Partido Socialista francés ${ }^{4}$. Para aquello, cruza entrevistas biográficas mediante las cuales busca reconstruir determinadas trayectorias militantes y dirigenciales, con observaciones minuciosas realizadas en diferentes sitios de implantación de este partido. Desde fines de los años ochenta, los trabajos enmarcados en esta perspectiva han recurrido al método etnográfico para observar in situ los grupos de interconocimiento que tejen la trama del espacio político local (Sawicki y Briquet, 1989) ${ }^{5}$.

Fundamentalmente, este enfoque permite comprender que las posiciones de poder que ocupan determinados actores en una organización partidaria deben a veces mucho a la densidad de sus vínculos sociales (profesionales, religiosos, asociativos, etc.) o a la(s) posición(es) que éstos ocupan en diferentes espacios políticos, sociales o laborales. El debate científico contemporáneo ha prestado mucha atención a los fenómenos de multiposicionalidad o de compromisos plurales, procesos sucesivos o concomitantes que implican la circulación de individuos, pero también de saberes y recursos que contribuyen a explicar las carreras militantes o dirigenciales. Es por ende el funcionamiento cotidiano de los partidos que esta sociología ha tomado como objeto central, postura coincidente con los anhelos de varios politólogos de la región (Luna y Rovira Kaltwasser, 2011: 19). Diferentes estudios sobre el compromiso plural levantan también la pregunta de las evoluciones contemporáneas de las formas de activismo y participación en la cosa pública, al mostrar la emergencia progresiva de otras relaciones entre el individuo, el espacio público y la esfera política (Ion, 2001).

Bajo el mismo enfoque etnográfico, Hélène Combes toma los cierres de campaña de los tres grandes partidos mexicanos (el PRI, el PAN y el PRD) en 2006 como "un espacio de

\footnotetext{
Véase también Sawicki (1997).

Entre las referencias más recientes destaca el libro editado por Javier Auyero et al. (2007).
} 
visualización del entorno partidista y de su evolución", articulando observación participante, entrevistas y la aplicación de una encuesta no aleatoria a militantes. El trabajo recurre a la sociología de los movimientos sociales para dar cuenta de la diversidad de sus formas de movilización (mediante organizaciones del entorno partidista, redes clientelares, el aparato territorial o/y más específicamente el trabajo de operadores de movilización). Asimismo, el efecto de vasos comunicantes que se produce entre las redes ciudadanas de la "sociedad civil" y los partidos estudiados confirma la importancia de tomar en consideración los fenómenos de multiposicionalidad y estudiar sus efectos sobre el campo partidista.

El trabajo de Bernard Pudal, elaborado a partir de una de sus ponencias dictadas en el marco de Jornadas Internacionales de Estudio sobre Militancia ${ }^{6}$, ofrece un marco muy útil para entender la evolución de "Los enfoques teóricos y metodológicos de la militancia". El autor destaca en particular el tránsito desde la tesis del "militante total" (cuyo referente es el activista obrero y militante comunista) al "militante distanciado" descrito y elogiado por Jacques Ion (1997), pasando por la "desacralización" del activismo obrero durante los años setenta y las críticas posteriores realizadas a la tesis de lon por no restituir toda la complejidad del compromiso. Según Pudal, la última etapa de las investigaciones sobre el tema se caracteriza por diferentes preocupaciones: pensar en términos de "construcción social de las causas"; dar cuenta de las lógicas diversas de la militancia y de diferentes tipos de militantes; estudiar las fases de desinvolucramiento para comprender mejor las fases de mayor intensidad del compromiso; interesarse por las "militancias olvidadas" tales como las militancias y activismos católicos o los involucramientos en asociaciones de voluntariado, etc. El artículo de Julien Fretel sobre la centro-derecha francesa (Unión para la Democracia Francesa-UDF y posterior Movimiento Demócrata-MoDem) participa de esta última generación de estudios.

Además de explicar cómo los partidos de derecha se convirtieron en objetos legítimos de investigación bajo el doble efecto del declive del modelo de partido "de masas" y de la "cartelización" de los sistemas de partidos (Katz y Mair, 1995), este artículo contribuye a derribar dos mitos: el que hace de las colectividades de centro-derecha partidos "de notables" y de la militancia una característica exclusiva de las formaciones de izquierda. Ambos supuestos son presentados por el autor como efectos colaterales de las tipologías que tendieron a medir la militancia y la institución partidista con la vara del partido "de masas" (Duverger, 1990 [1951]). Al contrario, Fretel muestra que los comportamientos "antiinstitucionales" de los dirigentes centristas (i.e. su distancia respecto del juego partidario o su "individualismo") reciben en realidad la influencia de una institución mucho más apremiante de lo que se suele creer. De la misma manera, la militancia "intermitente" o "laxa" que los caracteriza puede ser entendida a la luz de la relación con el partido y en contraste con otros tipos de involucramientos no partidarios (religiosos, cívicos y humanitarios) en el marco de los cuales los dirigentes adquieren "saberes en materia de acción colectiva", recursos y capitales. En este sentido, las carreras dirigenciales se enraízan en entornos partidistas

6 Estas Jornadas fueron organizadas por Daniela Cuadros en el marco del Proyecto ECOS-CONICYT C05H01. Véase www.conicyt.cl/documentos/dri/visitas/2007/Alenda.html 
que deben ser objetivados mediante una "etnografía multinivel", vale decir, el seguimiento de estos dirigentes en sus diferentes lugares de ejercicio del oficio político. En síntesis, el enfoque propuesto consiste en "entender cómo cada agrupación partidaria adquiere poco a poco su forma institucional y cómo sus actividades y sus actores se ordenan en ella, sea o no imponente su 'armazón' (...)". Esta "sociología de la institución", recientemente plasmada en un libro muy estimulante (Lagroye, Offerlé, 2011), preconiza restituir comparativamente "los principios de cada institucionalización" en vez de identificar propiedades genéricas abstractas de cada organización ${ }^{7}$.

Por último, la contribución de Daniel Grimaldi, doctorando en estudios políticos, tiene el mérito de sentar las primeras bases para comprender la evolución organizacional del Partido Por la Democracia (PPD) durante los veinte años de permanencia de la Concertación en el poder. Algunos de los resultados presentados, que provienen de una encuesta sociográfica aplicada en 2006 en el marco del Proyecto FONDECYT 1061034, dan cuenta de una "estatización" del personal partidista encuestado. Contribuyen asimismo a la reflexión sobre la profesionalización de los partidos políticos en Chile, mostrando de nuevo las dificultades de ajustar categorías abstractas (como la de "partido cartel") a la realidad de los objetos de estudio y a sus contextos de emergencia y desarrollo.

La idea de un número temático que reuniera diferentes generaciones de investigadores franceses con el fin de mostrar las convergencias y los aportes de sus enfoques sobre la militancia y la acción colectiva nació en el marco del Proyecto ECOS-CONICYT C05H01 (2006-2008). Quisiéramos agradecer al programa ECOS por haber financiado esta colaboración científica, al Director de la Revista de Sociología de la FACSO, Octavio Avendaño, por haber permitido que se materializara esta publicación, y al Doctorado en Ciencias Sociales de la Universidad de Chile por dar cabida a esta mirada sociológica sobre los fenómenos políticos, sin duda complementaria con los enfoques politológicos dominantes.

Santiago, octubre 2011.

\section{BIBLIOGRAFÍA}

Alenda, Stéphanie (2012): “La fabrique des leaders. Production, reproduction, transformation de l'Unión Demócrata Independiente-UDI chilienne (1965-2010)", en Jean-Gabriel Contamin, Bruno Duriez y Frédéric Sawicki (dirs.): Les continuités et discontinuités du militantisme Trajectoires, pratiques et organisations militantes, por publicarse.

Agrikoliansky, Eric, Olivier Fillieule y Nonna Mayer (dirs.) (2005): L'Altermondialisme en France. Genèse et dynamique d'un mouvement social, Flammarion, París.

Auyero, Javier, Lauren Joseph y Matthew Mahler (eds.) (2007): New Perspectives in Political Ethnography, Springer, New York.

7 Para una aplicación de este enfoque al caso de la Unión Demócrata Independiente chilena, véase Alenda (2012). 
Barrio Astrid et al. (2011): "Convention Delegates in Spain: State of the Art", ponencia presentada en el Workshop The current state of party member research, Copenhagen, 3 y 4 de febrero.

Boltanski, Luc (1973): “L'espace positionnel: multiplicité des positions institutionnelles et habitus de classe", Revue de sociologie française, 14 (1), pp. 3-20.

Collovald, Annie, Marie-Hélène Lechien, Sabine Rozier y Laurent Willemez (2003): L'humanitaire et le management des dévouements. Enquête sur un militantisme de "solidarité internationale" en faveur du Tiers Monde, Presses Universitaires de Rennes, Rennes.

Dalton, Russell J. y Martin P. Wattenberg (eds.) (2000): Parties without partisans. Political Change in Advanced Industrial Democracies, Oxford University Press, Oxford.

Della Porta, Donatella y Sidney Tarrow (2005): Transnational Protest and Global Activism, Rowman \& Littlefield Publishers, Lanham.

Duverger, Maurice (1990 [1951]): Les partis politiques, Armand Colin, París (Los partidos políticos, Fondo de Cultura Económica, México, 2006).

Gobille, Boris y Aysen Uysal (2005): "Cosmopolites et enracinés", en Isabelle Sommier y Eric Agrikoliansky (dirs.): Radiographie du mouvement altermondialiste, La Dispute, París, pp. 105-126.

Hipsher, Patricia (1996): "Democratization and the Decline of Urban Social Movements in Chile and Spain", Comparative Politics, 28 (3), pp. 273-297.

Ion, Jacques (1997): La fin des militants, Éditions de l'Atelier, París.

(2001): L'Engagement au pluriel, Publications de l'Université de Saint-Etienne, Saint-Etienne.

Katz, Richard y Peter Mair (1995): “Changing Modes of Party Organization and Party Democracy: the Emergence of the Cartel Party", Party Politics, 1 (1), pp. 5-28.

Lagroye, Jacques y Michel Offerlé (dirs.) (2011): Sociologie de l'institution, Belin, París.

Luna, Juan Pablo y Cristóbal Rovira Kaltwasser (2011): “Las derechas gobernantes en América Latina: hacia una caracterización preliminar", LASA Forum, XLII (3), pp. 16-19.

McAdam, Doug (1982): Political Process and the Development of Black Insurgency, 1930-1970, University of Chicago Press, Chicago.

Offerlé, Michel (1987): Les partis politiques, Presses Universitaires de France, París (Los partidos políticos, LOM, Santiago, 2004).

Oxhorn, Philip (1994): "Where did All the Protesters Go?: Popular Mobilization and the Transition to Democracy in Chile", Latin American Perspectives, 21 (3), pp. 49-68.

Rocha, Daniella (2009): “Militantismo partidário e experiência de poder. O caso do PT no Distrito Federal", Antropolítica (UFF), 23, pp. 67-95.

Sawicki, Frédéric (1997): Les réseaux du parti socialiste: sociologie d'un milieu partisan, Belin, París.

Sawicki, Frédéric y Jean-Louis Briquet (1989): "L’analyse localisée du politique”, Politix, 2 (7-8), pp. 6-16.

Schneider, Cathy Lisa (2000): "Violence, Identity, and Spaces of Contention in Chile, Argentina and Colombia", Social Research, 67 (3), pp. 773-802.

Tarrow, Sidney (2000): "La contestation transnationale", Cultures et Conflits, 38-39, pp. 197-223.

Torcal, Mariano (2003): "Political Disaffection and Democratization History in New Democracies", Working Paper, 308, Kellogg Institute. 